\title{
Systematische Untersuchung der Azikularferritbildung in Stählen
}

\author{
Denise Loder, Susanne Katharina Michelic und Christian Bernhard \\ Lehrstuhl für Eisen- und Stahlmetallurgie, Montanuniversität Leoben, Leoben, Österreich
}

Eingegangen 21. April 2016; angenommen 25. April 2016; online publiziert 28. Juni 2016

\begin{abstract}
Zusammenfassung: Die Azikularferritbildung ist durch die Stahlzusammensetzung, Kühlrate, Austenitkorngröße und Einschlusslandschaft bestimmt, jedoch ist deren exakte Wirkung noch nicht restlos geklärt. Im Rahmen des FWFProjekts TRP 266-N19 wurde eine systematische Methodik zur Untersuchung dieser Einflussfaktoren sowie deren Wechselwirkungen entwickelt. Die vorliegende Arbeit demonstriert die Anwendung dieser Methodik beispielhaft für die Untersuchung des Einflusses von Kühlrate und Bor auf die Azikularferritbildung in einem niederkohligen HSLAStahl.
\end{abstract}

Schlüsselwörter: Azikularer Ferrit, HSLA-Stahl, Nichtmetallische Einschlüsse, Mikrostruktur

Systematic Study of the Acicular Ferrite Formation in Steels

Abstract: The formation of acicular ferrite is mainly influenced by the steel composition, cooling rate, austenite grain size, and inclusion landscape. However, their precise effects have not been clarified in literature yet. Within the FWF project TRP 266-N19, a systematic methodology to study the influencing factors of the acicular ferrite formation as well as their interactions on laboratory scale was developed. The present work exemplarily demonstrates the application of this methodology for studying the effect of cooling rate and boron on the formation of acicular ferrite in a low-carbon HSLA steel.

Keywords: Acicular ferrite, HSLA steel, Non-metallic inclusions, Microstructure

D. Loder ( $\square)$

Lehrstuhl für Eisen- und Stahlmetallurgie,

Montanuniversität Leoben,

Franz-Josef-Straße 18,

8700 Leoben, Österreich

E-Mail: denise.loder@unileoben.ac.at

\section{Einleitung}

Azikularferrit (AF) keimt im Stahl intragranular an nichtmetallischen Einschlüssen (NME) und bildet ein Gefüge aus feinen, ineinandergreifenden Körnen [1-3]. Diese charakteristische Mikrostruktur kann eine deutliche Verbesserung der Zähigkeit bewirken [4-6]. Zusätzlich zeigt azikularer Ferrit eine ausgezeichnete Beständigkeit gegen $\mathrm{H}_{2} \mathrm{~S}$-Angriff [7]. Aufgrund dieser Eigenschaften hat in den letzten Jahren die Verwendung von Azikularferrit in Röhrenstählen deutlich an Bedeutung gewonnen [5-9]. Es ist bekannt, dass die Bildung von azikularem Ferrit primär durch die Stahlzusammensetzung, Einschlusslandschaft, Kühlrate und Austenitkorngröße bestimmt wird, jedoch ist die Wechselwirkung dieser Faktoren noch nicht restlos geklärt.

Im Rahmen eines FWF-Projekts am Lehrstuhl für Eisenund Stahlmetallurgie wurde eine systematische Methodik zur Untersuchung der Azikularferritbildung und deren Einflussfaktoren im Labormaßstab entwickelt. Die Methodik verwendet nur Einschlüsse resultierend aus Desoxidation und Entschwefelung als Keimstellen für Azikularferrit. Damit kann eine künstliche Verschlechterung des Stahlreinheitsgrads durch die Zugabe synthetischer Partikel vermieden werden. Mit Hilfe dieser Methodik wurde die Azikularferritbildung in nieder-, mittel- und hochkohligen Stahlgüten ausführlich untersucht. Besonderer Fokus wurde dabei auf die Untersuchung aktiver Einschlusstypen gelegt.

Der vorliegende Beitrag gibt einen Überblick über die entwickelte Methodik und demonstriert die Anwendung dieser beispielhaft für die Bestimmung des Einflusses von Kühlrate und Bor-Gehalt in einem niederkohligen HSLAStahl. Die Literatur zeigt, dass gelöstes Bor an Austenitkorngrenzen seigert, wodurch deren Eignung als Keimstelle für Ferrit reduziert und die intragranulare Keimung von azikularem Ferrit begünstigt wird [10-14]. Bor seigert auch an den Grenzflächen zu nichtmetallischen Einschlüssen, jedoch können ausgewählte Einschlusstypen, z.B. $\mathrm{TiO}_{x}$, Bor aufnehmen und eine B-verarmte Zone ausbilden, so dass das Keimungspotential dieser Einschlüsse nicht reduziert wird [11]. Der Einfluss von Bor auf die Bildung von Azikularferrit 
in Schweißnähten mit ungefähr 0,07 Gew.-\% Kohlenstoff, 1,50 Gew.-\% Mangan, 0,40 Gew.-\% Silizium, 2 - 660 Gew.ppm Titan und 1 - 195 Gew.-ppm Bor wurde bereits von Evans [10] und IIman et al. [13] untersucht. Dabei stellten sie fest, dass geringe Mengen an Bor dienlich für die Steigerung des Azikularferritgehalts sind, größere Mengen jedoch einen negativen Effekt haben, da es zur Bildung von $\mathrm{Fe}_{23}(\mathrm{BC})_{6}$-Layern auf aktiven Einschlüssen kommt. Der kritische Bor-Gehalt hängt stark vom Ti-Gehalt $a b$, für einen Ti-Gehalt von 0,02 Gew.-\% liegt dieser bei ca. 50 Gew.-ppm Bor. Der positive Einfluss von B-Gehalten unter 40 Gew.ppm konnte kürzlich auch durch Zhang et al. [3] für mittelkohlige Stähle mit 0,15 Gew.-\% Kohlenstoff, 1,50 Gew.-\% Mangan, 0,20 Gew.-\% Silizium, 100 Gew.-ppm Titan und 2 - 33 Gew.-ppm Bor mittels HT-LSCM-Untersuchungen bestätigt werden. Aufbauend auf den Erkenntnissen aus der Literatur untersucht die vorliegenden Arbeit, ob ein ähnliches Verhalten von Bor auch in niederkohligen HSLAStählen mit 0,04 Gew.-\% Kohlenstoff und ohne erneutes Aufschmelzen des Materials auftritt. Hierzu wird die Azikularferritbildung während einer Wärmebehandlung ohne Umformschritt simuliert. Die erzielten Ergebnisse können daher nicht mit jenen einer thermomechanischen Behandlung verglichen werden.

\section{Methodik}

Tab. 1 zeigt die chemische Zusammensetzung des untersuchten niederkohligen HSLA-Stahls mit variierendem Bor-Gehalt. Die untersuchte Stahlzusammensetzung ist an Röhrenstähle für den Pipelinebau angelehnt, weist jedoch einen höheren Schwefelgehalt als typische Röhrenstähle auf. Die Untersuchung der Azikularferritbildung erfolgt mit einer vierstufigen Methodik (siehe Abb. 1).

Im ersten Schritt der Methodik erfolgt die Simulation der Einschlussbildung und -modifikation während der Tammannofenversuche mittels FactSage 6.4 unter Verwendung der Datenbanken FactPS, FToxid und FSstel. Einschlussdiagramme stellen die stabilen Einschlusstypen in Abhängigkeit zweier Legierungselemente dar und ermöglichen so durch Adaptierung der Stahlzusammensetzung die gezielte Einstellung spezifischer Einschlusstypen. Abb. 2 zeigt das Sauerstoff-Schwefel-Einschlussdiagramm für Stahl A. Um sowohl $\mathrm{TiO}_{x}$ als auch ASlag, eine homogene Mischung oxidischer und sulphidischer Phasen, hinsichtlich ihrer Wirkung als Keimstellen für Azikularferrit untersuchen zu können, wird ein Ausgangssauerstoffgehalt von 125 Gew.-ppm ( $\log O=-1,9$ Gew.-\%) eingestellt. Das verwendete Grundmaterial für die Schmelzversuche enthält bereits $80 \mathrm{Gew}$.ppm Schwefel ( $\log S=-2,1$ Gew.-\%). Auf eine weitere Zu- gabe von Schwefel wurde verzichtet. Laut Einschlussdiagramm für $1500^{\circ} \mathrm{C}$ ist $\mathrm{MnS}$ bei diesem Schwefelgehalt noch nicht stabil. Jedoch ist zu beachten, dass sich die MnS-Stabilitätslinie mit sinkender Temperatur zu niedrigeren S-Gehalten verschiebt, so dass sich mit fortschreitender Kühlung MnS auch bei 80 Gew.-ppm S ausscheidet. Die ermittelte Stahlzusammensetzung wird als Input für ein Prozessmodell verwendet, welches schrittweise den Schmelzversuch im Tammannofen simuliert. Abschließend wird die Veränderung der Einschlusslandschaft während der Erstarrung und Kühlung berechnet.

Im zweiten Schritt werden Proben in einem Tammannofen erschmolzen. Besonderes Augenmerk wird dabei auf die Erzeugung einer homogen verteilten Einschlusslandschaft durch regelmäßiges Rühren und eine beschleunigte Abkühlung gelegt. Schritt drei stellt die Wärmebehandlung der erzeugten Stähle im Hochtemperatur-LaserscanningKonfokalmikroskop (HT-LSCM) dar. Der große Vorteil dieses Geräts ist die Möglichkeit, die Keimung und das Wachstum von azikularen Ferritnadeln an nichtmetallischen Einschlüssen in situ beobachten zu können, wie in (Abb. 3) beispielhaft zu sehen.

Abschließend erfolgt die metallographische Analyse von Azikularferritgehalt, Austenitkorngröße und Einschlusslandschaft bestehend aus Anzahl, Größe, Typ und Morphologie nichtmetallischer Einschlüsse. Für die Bewertung von Azikularferritgehalt und Austenitkorngröße finden semi-automatisierte Auswerteroutinen Verwendung. Die Charakterisierung von nichtmetallischen Einschlüssen erfolgt mittels REM/EDX-Analysen. Beispielhaft ist in Abb. 3 ein aktiver ( $\mathrm{Ti}, \mathrm{Mn}, \mathrm{Al}) \mathrm{O}_{\mathrm{x}} \mathrm{S}_{\mathrm{y}}$ Einschluss mit zwei angekeimten Azikularferritnadeln gezeigt.

Weitere Informationen zur entwickelten Methodik sind in Veröffentlichungen der Arbeitsgruppe zu finden [15-18].

\section{Einfluss von Kühlrate und Bor auf die Azikularferritbildung}

Der Einfluss der Kühlrate ist in Abb. 4 dargestellt. Exemplarisch ist das Gefüge in Stahl A für eine mittlere Kühlrate von $-200^{\circ} \mathrm{C} / \mathrm{min}$ und eine langsame Kühlrate von $-20^{\circ} \mathrm{C} / \mathrm{min}$ zwischen 800 und $500{ }^{\circ} \mathrm{C}$ gezeigt. Beide Proben bestehen zum Großteil aus Bainit, mit geringeren Mengen an Azikularferrit. Es ist deutlich zu erkennen, dass trotz der starken Variation in der Kühlrate nur geringe Änderungen im Gefüge auftreten.

Wie in Abb. 5 zu sehen ist, erhöht die Zugabe von Bor den Anteil an azikularem Ferrit im Gefüge. Durch 12 Gew.ppm B steigt der Azikularferritgehalt von 8 auf $14 \%$. Wird der B-Gehalt weiter auf $33 \mathrm{Gew}$.-ppm angehoben, sinkt der

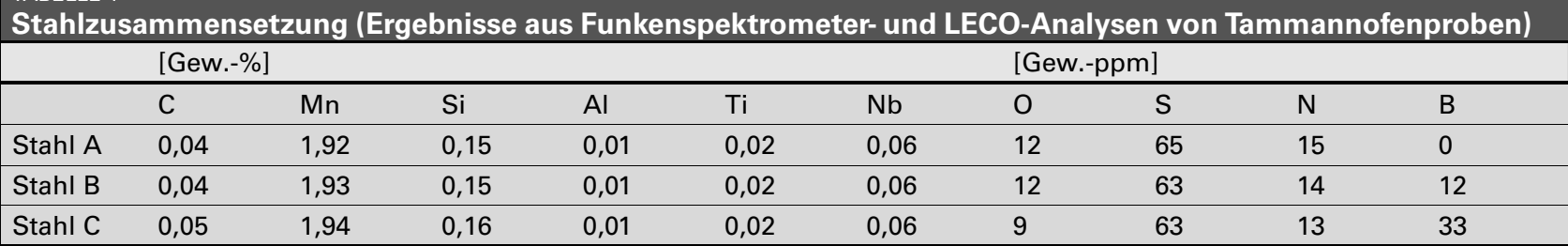


Abb. 1: Systematische Methodik zur Untersuchung der Azikularferritbildung im Labormaßstab

Abb. 2: O-S-Einschlussdiagramm Stahl A
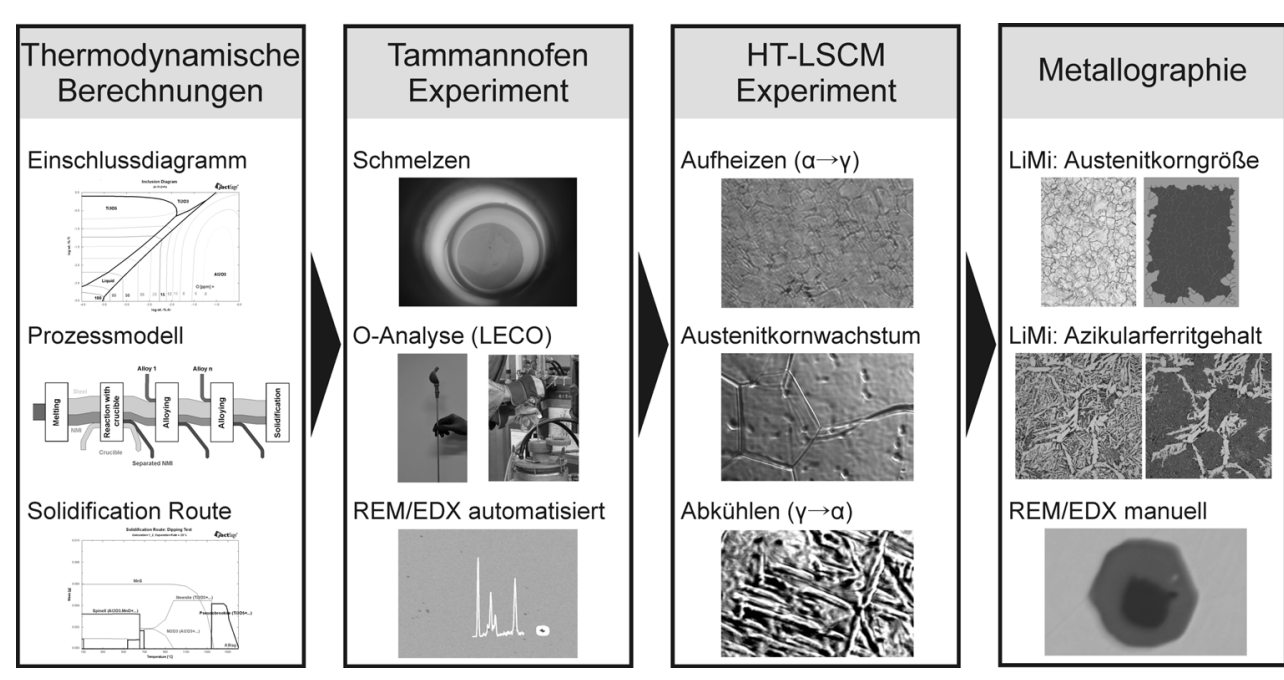

Einschlussdiagramm Stahl A $0,04 \mathrm{C}-1,90 \mathrm{Mn}-0,15 \mathrm{Si}-0,02 \mathrm{Ti}-0,06 \mathrm{Nb}-0,003 \mathrm{~N} ; 1500{ }^{\circ} \mathrm{C}$

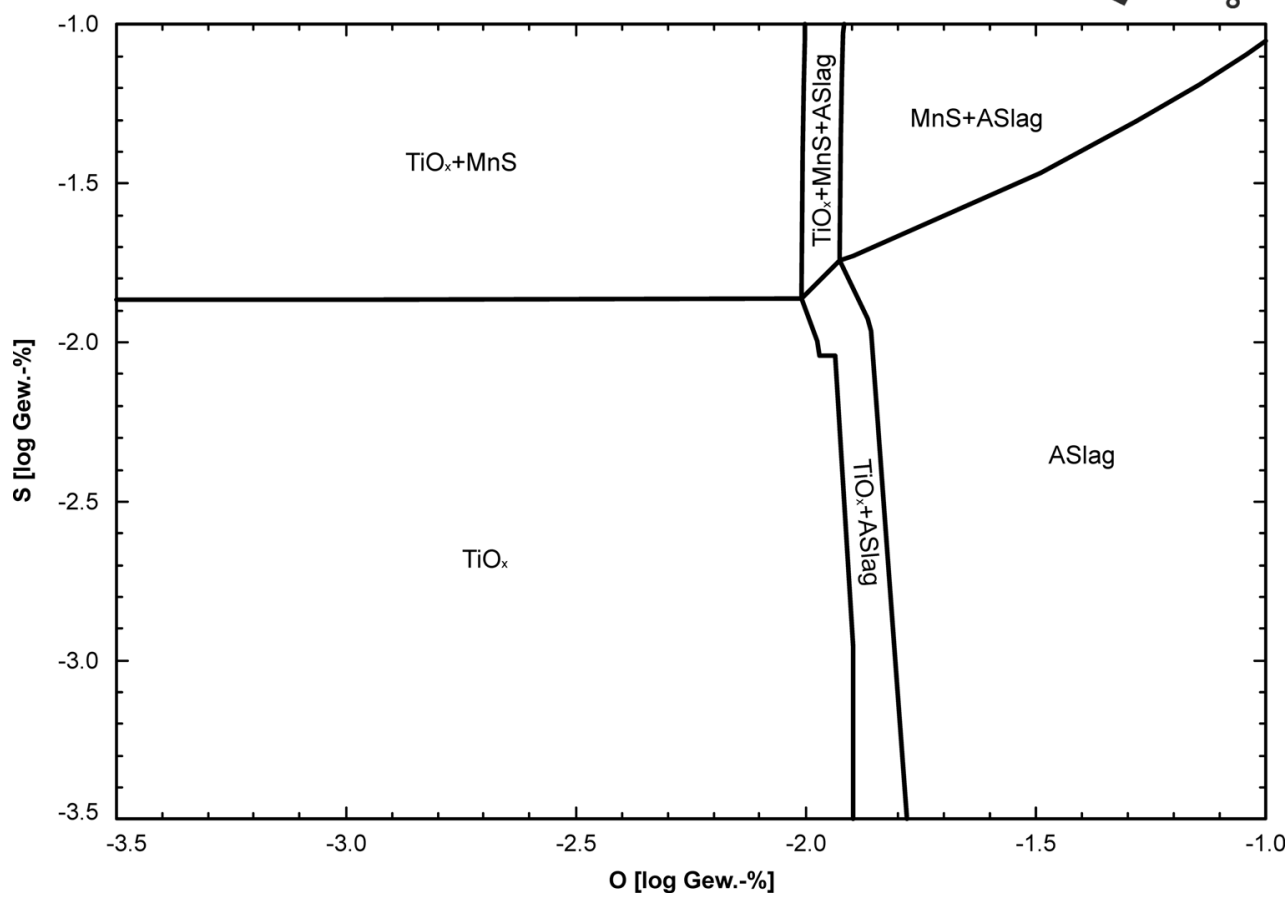

Azikularferritanteil wieder auf $10 \%$. Es wird vermutet, dass dies mit der in der Literatur $[10,13]$ beschriebenen Inaktivierung von Einschlüssen durch $\mathrm{Fe}_{23}(\mathrm{BC})_{6}$-Layer zusammenhängt.

Auch in den B-legierten Güten konnte kein signifikanter Einfluss der Kühlrate beobachtet werden. Wie in Abb. 5 für Stahl B gezeigt, verändert sich der Anteil an azikularem Ferrit bei Variation der Kühlrate nicht. Folglich ist die Kühlrate zwar eine essentielle Voraussetzung für die Azikularferritbildung, aber die Menge an Azikularferrit wird im vorliegenden System kaum von der Kühlrate beeinflusst. Es soll jedoch angemerkt werden, dass weitere Untersuchungen in diesem Projekt $[16,18,19]$ zeigten, dass der KühIrateneinfluss stark von der Stahlgüte und dem Herstellprozess abhängt.

\section{Zusammenfassung}

Die vorliegende Arbeit beschreibt eine systematische Methodik im Labormaßstab zur Untersuchung der Azikularferritbildung in Stählen und deren Einflussfaktoren. Die Methodik wird beispielhaft für die Analyse des Einflusses von Kühlrate und Bor auf den Azikularferritgehalt in niederkohligen HSLA-Stählen angewandt. Dabei konnten folgende Erkenntnisse gewonnen werden:

1. Obwohl die Kühlrate wesentliche Voraussetzung für die Bildung von Azikularferrit ist, ist eine Optimierung des Azikularferritgehalts im untersuchten HSLA-Stahl über die Kühlrate nur schwer möglich, da in dieser Güte der 
Abb. 3: In situ Beobachtung der Azikularferritbildung in Stahl A-1 mittels HT-LSCM und aktiver (Ti,Mn,Al) $\mathrm{O}_{x} \mathrm{~S}_{y}$ Einschluss

Abb. 4: Einfluss der Kühlrate auf den Azikularferritgehalt in Stahl A
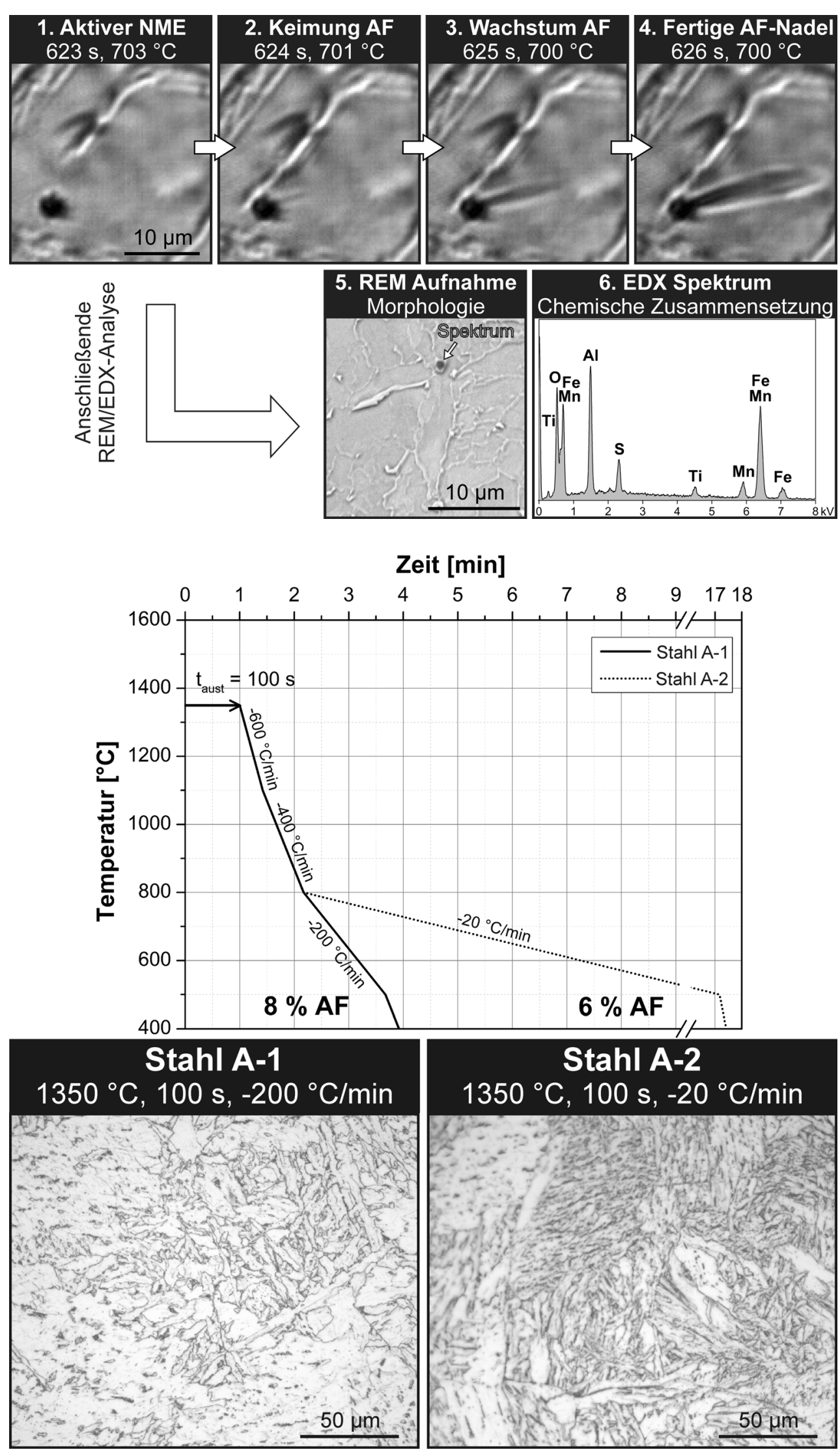
Abb. 5: Einfluss von Bor (B) und Kühlrate (CR) auf den Azikularferritgehalt

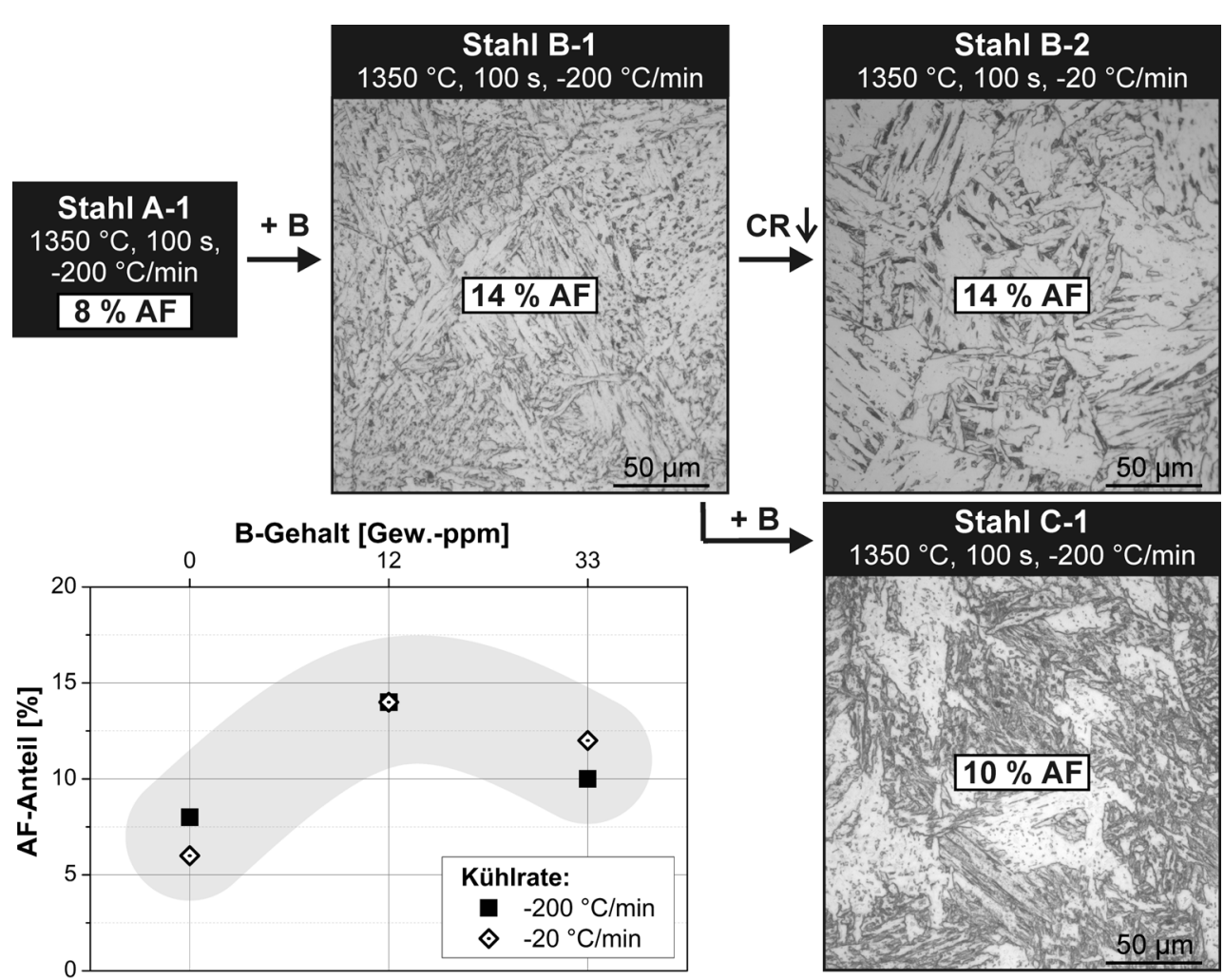

Anteil an azikularem Ferrit über weite Kühlratenbereiche nur geringe Änderungen zeigt.

2. Bor-Zugaben von $<30$ Gew.-ppm erhöhen den Azikularferritgehalt, bei größeren Zugaben tritt ein gegenteiliger Effekt auf.

3. Der Effekt von Bor auf die Azikularferritbildung hängt nicht von der Herstellroute (Austenitisierung oder Schweißen) ab.

\section{Danksagung}

Die Autoren danken für die finanzielle Unterstützung des Bundesministeriums für Verkehr, Innovation und Technology (bmvit) und dem Österreichischen Wirtschaftsfonds (FWF) im Rahmen des Projekts TRP 266-N19.

Open access funding provided by Montanuniversität Leoben.

Open Access Dieser Artikel wird unter der Creative Commons Namensnennung 4.0 International Lizenz (http://creativecommons.org/licenses/by/4.0/deed.de) veröffentlicht, welche die Nutzung, Vervielfältigung, Bearbeitung, Verbreitung und Wiedergabe in jeglichem Medium und Format erlaubt, sofern Sie den/die ursprünglichen Autor(en) und die Quelle ordnungsgemäß nennen, einen Linkzur Creative Commons Lizenz beifügen und angeben, ob Änderungen vorgenommen wurden.

\section{Literatur}

1. Díaz-Fuentes, M.; Iza-Mendia, A.; Gutiérrez, I.: Analysis of different acicular ferrite microstructures in low-carbon steels by electron backscattered diffraction. Study of their toughness behavior,
Metallurgical and Materials Transactions A, 34 (2003), Nr. 11, S. 2505-2516

2. Wan, X. L.; Wang, H. H.; Cheng, L.; Wu, K. M.: The formation mechanisms of interlocked microstructures in low-carbon high-strength steel weld metals, Materials Characterization, 67 (2012), S. 41-51

3. Zhang, D.; Shintaku, Y.; Suzuki, S.; Komizo, Y. I.: In situ observation of phase transformation in low-carbon, boron-treated steels, Metallurgical and Materials Transactions A, 43 (2012), Nr. 2, S. 447-458

4. Eijk, C. van der; Grong, Ø.; Hjelen, J.: Quantification of inclusionstimulated ferrite nucleation in wrought steel using the SEM-EBSD technique, in: Koiwa, M.; Otsuka, K.; Miyazaki, T. (Hrsg.): Proceedings of the International Conference on Solid-Solid Phase Transformations, Kyoto, Japan, 1999, S. 1573-1576

5. Zhao, M. C.; Yang, K.; Shan, Y. Y.: Comparison on strength and toughness behaviors of microalloyed pipeline steels with acicular ferrite and ultrafine ferrite, Materials Letters, 57 (2003), Nr. 9-10, S. 1496-1500

6. Wu, X.; Lee, H.; Kim, Y. M.; Kim, N. J.: Effects of processing parameters on microstructure and properties of ultra high strength linepipe steel, Journal of Materials Science and Technology, 28 (2012), Nr. 10, S. 889-894

7. Zhao, M. C.; Shan, Y. Y.; Xiao, F. R.; Yang, K.; Li, Y. H.: Investigation on the $\mathrm{H}_{2} \mathrm{~S}$-resistant behaviors of acicular ferrite and ultrafine ferrite, Materials Letters, 57 (2002), Nr. 1, S. 141-145

8. Xiao, F.; Liao, B.; Ren, D.; Shan, Y.; Yang, K.: Acicular ferritic microstructure of a low-carbon $\mathrm{Mn}-\mathrm{Mo}-\mathrm{Nb}$ microalloyed pipeline steel, Materials Characterization, 54 (2005), Nr. 4-5, S. 305-314

9. Kim, Y. M.; Lee, H.; Kim, N. J.: Transformation behavior and microstructural characteristics of acicular ferrite in linepipe steels, Materials Science and Engineering A, 478 (2008), Nr. 1-2, S. 361-370

10. Evans, G. M.: Microstructure and properties of ferritic steel welds containing Ti and B, Welding Research Supplement, (1996), S. 251s-260s

11. Yamamoto, K.; Hasegawa, T.; Takamura, J. I.: Effect of boron on in tra-granular ferrite formation in Ti-Oxide bearing steels, ISIJ International, 36 (1996), Nr. 1, S. 80-86 
12. Peng, Y.; Chen, W.; Xu, Z.: Study of high toughness ferrite wire for submerged arc welding of pipeline steel, Materials Characterization, 47 (2001), Nr. 1, S. 67-73

13. IIman, M. N.; Cochrane, R. C.; Evans, G. M.: Effect of nitrogen and boron on the development of acicular ferrite in reheated C-Mn-Ti steel weld metals, Welding in the World, 56 (2012), Nr. 11-12, S. 41-50

14. Ilman, M. N.; Cochrane, R. C.; Evans, G. M.: The development of acicular ferrite in reheated Ti-B-Al-N type steel weld metals containing various levels of aluminium and nitrogen, Welding in the World, 59 (2015), Nr. 4, S. 565-575

15. Loder, D.; Michelic, S. K.; Bernhard, C.: Characterization of acicular ferrite microstructures using etching methods, optical microscopy and HT-LSCM, in: Mayer, S.; Panzenböck, M.; Clemens, H. (Hrsg.): Fortschritte in der Metallographie 46, Berichte der 14. Internationalen Metallographie-Tagung, Leoben, Österreich, 2014, S. 125-130
16. Loder, D.; Michelic, S. K.; Mayerhofer, A.; Bernhard, C.; Dippenaar, R. J.: In situ observation of acicular ferrite formation using HTLSCM: possibilities, challenges and influencing factors, in: Proceedings of MS\&T Conference, Pittsburgh, USA, 2014, S. 469-476

17. Loder, D; Michelic, S. K.: Specific use of non-metallic inclusions for the formation of acicular ferrite structures: Thermodynamic modeling and laboratory experiments, Proceedings of the 9th International Conference on Clean Steel, Budapest, Ungarn, 2015, S. 1-12

18. Loder, D; Michelic, S. K.: Systematic investigation of acicular ferrite formation on laboratory scale, Materials Science and Technology, (2016), S. 1-10

19. Michelic, S. K.; Loder, D.; Arth, G.; Bernhard, C.: Experimental study on the formation of non-metallic inclusions acting as nuclei for acicular ferrite in HSLA steels through specific deoxidation practice and defined cooling conditions, Materials Science Forum, 783-786 (2014), S. 1079-1084 